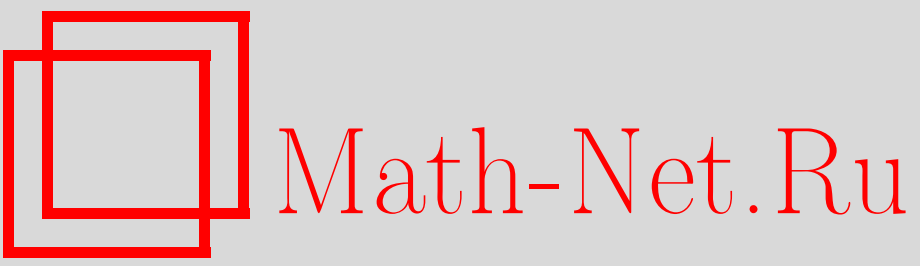

П. А. Вельмисов, А. В. Анкилов, Ю. В. Покладова, Математическое моделирование вибрационных устройств, Итоги науки и техн. Сер. Соврем. мат. и ее прил. Темат. обз., 2020, том 185, 37-49

DOI: https://doi.org/10.36535/0233-6723-2020-185-37-49

Использование Общероссийского математического портала Math-Net.Ru подразумевает, что вы прочитали и согласны с пользовательским соглашением

http://www.mathnet.ru/rus/agreement

Параметры загрузки:

IP : 3.81 .55 .215

26 апреля 2023 г., 08:16:52 


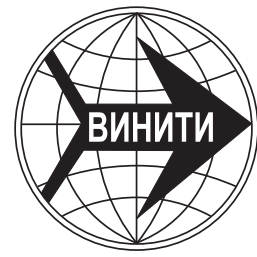

ИТОГИ НАУКИ И ТЕХНИКИ.

Современная математика и ее приложения.

Тематические обзоры.

Том 185 (2020). С. $37-49$

DOI: $10.36535 / 0233-6723-2020-185-37-49$

УДК $517.9 ; 532.5 ; 539.3$

\title{
МАТЕМАТИЧЕСКОЕ МОДЕЛИРОВАНИЕ ВИБРАЦИОННЫХ УСТРОЙСТВ
}

\author{
(c) 2020 г. П. А. ВЕЛЬМИСОВ, А. В. АНКИЛОВ, Ю. В. ПОКЛАДОВА
}

\begin{abstract}
АннотАция. Рассматриваются математические модели вибрационных устройств, предназначенных для интенсификации технологических процессов. Математические модели представляют собой начально-краевые задачи для связанных систем дифференциальных уравнений с частными производными для гидродинамических функций и функций деформаций упругих элементов. Исследуется динамика и динамическая устойчивость упругих элементов. Исследование динамики осуществляется на основе метода Бубнова-Галеркина. Исследование динамической устойчивости проводится на основе построения положительно определенных функционалов типа Ляпунова.

Ключевые слова: аэрогидроупругость, упругая пластина, деформация, динамика, устойчивость, дифференциальное уравнение с частными производными, метод Бубнова-Галеркина, функционал Ляпунова.
\end{abstract}

\section{MATHEMATICAL MODELING OF VIBRATION DEVICES}

\author{
(C) 2020 P. A. VELMISOV, A. V. ANKILOV, YU. V. POKLADOVA
}

\begin{abstract}
Mathematical models of vibration devices designed to intensify technological processes are considered. Mathematical models are initial-boundary-value problems for coupled systems of partial differential equations for hydrodynamic functions and deformation functions of elastic elements. We examine the dynamics and dynamic stability of elastic elements. The study of dynamics is based of the Bubnov-Galerkin method. The study of dynamical stability is based on the construction of positive definite Lyapunov-type functionals.
\end{abstract}

Keywords and phrases: aerohydroelasticity, elastic plate, deformation, dynamics, stability, partial differential equation, Bubnov-Galerkin method, Lyapunov functional.

AMS Subject Classification: $74 \mathrm{~F} 10$

1. Введение. При проектировании и эксплуатации конструкций, приборов, устройств, установок различного назначения, взаимодействующих с потоком газа или жидкости, важной проблемой является обеспечение надежности их функционирования и увеличение сроков службы. Подобные проблемы присущи многим отраслям техники. В частности, такого рода задачи возникают в авиаракетостроении, приборостроении, при проектировании антенных установок, высоких наземных сооружений и т. Д. Существенное значение при расчете конструкций, взаимодействующих с потоком газа, имеет исследование устойчивости деформируемых элементов, так как воздействие потока может приводить к ее потере. В качестве примеров потери динамической устойчивости можно указать: изгибно-крутильный флаттер крыла самолета; панельный флаттер

Работа выполнена при поддержке Российского фонда фундаментальных исследований и Правительства Ульяновской области (проект № 18-41-730015). 
пластин и оболочек, обтекаемых потоком, например флаттер панели обшивки самолета или ракеты; срывной флаттер лопаток турбин и винтов; колебания проводов, дымовых труб, висячих мостов и т. д.

В то же время для функционирования некоторых технических устройств явление возбуждения колебаний при аэрогидродинамическом воздействии, указанное выше в качестве негативного, является необходимым. Примерами подобных устройств, относящихся к вибрационной технике, являются устройства, используемые для интенсификации технологических процессов. Например, устройства для приготовления однородных смесей и эмульсий, в частности, установок для подачи смазочно-охлаждающей жидкости в зону обработки (см., например, [11]). Другим примером являются датчики давления. В этом случае деформация чувствительного элемента датчика необходима для функционирования приборов [13].

Основной частью широкого класса подобных устройств является проточный канал, на стенках которого (или внутри него) расположены упругие элементы. Работа таких устройств основана на вибрации упругих элементов при протекании внутри каналов жидкости.

Таким образом, при проектировании конструкций и устройств, находящихся во взаимодействии с потоком газа, необходимо решать задачи, связанные с исследованием устойчивости, требуемой для их функционирования и надежности эксплуатации.

Устойчивости упругих тел, взаимодействующих с потоком газа, посвящено большое количество теоретических и экспериментальных исследований, проведенных в последние десятилетия. Среди последних исследований по динамике и устойчивости деформируемых тел, обтекаемых потоком жидкости или газа, следует отметить работы $[1-3,15-18,21-26]$ и многие другие. Среди работ авторов данной статьи по исследованию динамики и устойчивости упругих тел, взаимодействующих с потоком жидкости или газа, отметим монографии и статьи $[4-10,12,19,20]$.

Принятые в работе определения устойчивости упругого тела соответствуют концепции устойчивости динамических систем по Ляпунову. Проблема может быть сформулирована так: при каких значениях параметров, характеризующих систему «жидкость-тело» (основными параметрами являются скорость потока, прочностные и инерционные характеристики тела, сжимающие или растягивающие усилия, силы трения), малым деформациям тел в начальный момент времени $t=0$ (т.е. малым начальным отклонениям от положения равновесия) будут соответствовать малые деформации и в любой момент времени $t>0$.

В статье рассматривается математическая модель гидродинамического излучателя - вибрационного устройства, предназначенного для приготовления однородных смесей и эмульсий. Основным компонентом устройства является упругий элемент, расположенный в проточном канале. Колебания упругого элемента приводят к размешиванию неоднородной среды, подаваемой в этот канал.

2. Постановка задачи. Рассматривается плоская задача аэрогидроупругости о малых колебаниях, возникающих при дозвуковом обтекании упругой пластины потенциальным потоком газа или жидкости в канале $G=\left\{(x, y) \in \mathbb{R}^{2}: 0<x<l, 0<y<h\right\}$ с прямолинейными стенками $y=0, y=h, 0<x<l$ (рис. 1). Скорость потока равна $V$ и направлена вдоль оси $O x$. Продольный и поперечный размеры канала равны $l, h$. Пластина в недеформированном состоянии занимает положение $y=h_{*}, a<x<b$.

Математическая постановка задачи имеет следующий вид:

$$
\begin{array}{ll}
\varphi_{x x}+\varphi_{y y}=0, & x \in(0, l), y \in(0, h), \\
\varphi(0, y, t)=\varphi(l, y, t)=0, & y \in(0, h), \\
\varphi_{y}(x, 0, t)=\varphi_{y}(x, h, t)=0, & x \in(0, l), \\
\varphi_{y}^{ \pm}\left(x, h_{*}, t\right)=w_{t}+V w_{x}, & x \in(a, b), \\
w(a, t)=w_{x x}(a, t)=0, w(b, t)=w_{x x}(b, t)=0, & t \geqslant 0, \\
L(w)=\rho\left(\varphi_{t}^{+}+V \varphi_{x}^{+}\right)-\rho\left(\varphi_{t}^{-}+V \varphi_{x}^{-}\right), & x \in(a, b),
\end{array}
$$




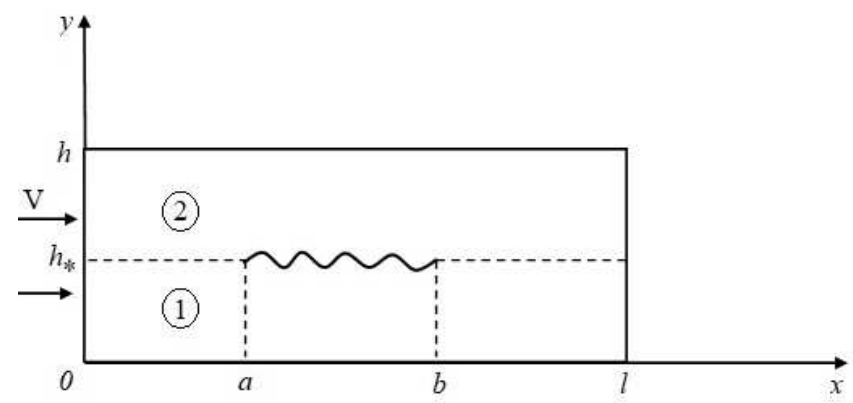

Рис. 1. Проточный канал с одним упругим элементом

где

$$
L(w) \equiv D w_{x^{4}}(x, t)+m w_{t t}(x, t)+N(t) w_{x x}(x, t)+\beta_{2} w_{x^{4} t}(x, t)+\beta_{1} w_{t}(x, t)+\beta_{0} w(x, t) .
$$

Здесь $w(x, t), x \in(a, b)$ - деформация упругой пластины в направлении оси $O y ; \varphi(x, y, t)$ - функция, определяющая потенциал скорости возмущенного потока газа; $g^{ \pm}=\lim _{y \rightarrow h_{*} \pm 0} g ;$ индексы $x, y, t$ снизу обозначают производные по $x, y, t ; m-$ погонная масса пластины; $D$ - изгибная жесткость пластины; $N(t)$ - сжимающая $(N>0)$ или растягивающая $(N<0)$ пластину сила; $\beta_{2}, \beta_{1}$ - коэффициенты внешнего и внутреннего демпфирования; $\beta_{0}$ - коэффициент жесткости основания (постели); $V, \rho$-скорость, плотность газа в однородном потоке.

3. Исследование устойчивости. Исследуется устойчивость нулевого решения $\varphi(x, y, t) \equiv 0$, $w(x, t) \equiv 0$ системы $(1)-(7)$ по отношению к возмущениям начальных данных. Рассмотрим функционал

$$
\begin{array}{r}
\Phi(t)=\iint_{G}\left(\varphi_{x}^{2}(x, y, t)+\varphi_{y}^{2}(x, y, t)\right) d x d y+2 V \int_{a}^{b}\left(\varphi^{+}\left(x, h_{*}, t\right)-\varphi^{-}\left(x, h_{*}, t\right)\right) w_{x}(x, t) d x+ \\
+\frac{1}{\rho} \int_{a}^{b}\left(m w_{t}^{2}(x, t)+D w_{x x}^{2}(x, t)-N(t) w_{x}^{2}(x, t)+\beta_{0} w^{2}(x, t)\right) d x .
\end{array}
$$

Найдем производную от $\Phi(t)$ по $t$ :

$$
\begin{aligned}
\Phi_{t}(t)=2 \iint_{G}\left(\varphi_{x} \varphi_{x t}+\right. & \left.\varphi_{y} \varphi_{y t}\right) d x d y+2 V \int_{a}^{b}\left(\varphi_{t}^{+}\left(x, h_{*}, t\right)-\varphi_{t}^{-}\left(x, h_{*}, t\right)\right) w_{x}(x, t) d x+ \\
& +2 V \int_{a}^{b}\left(\varphi^{+}\left(x, h_{*}, t\right)-\varphi^{-}\left(x, h_{*}, t\right)\right) w_{x t}(x, t) d x+ \\
& +\frac{2}{\rho} \int_{a}^{b}\left(m w_{t} w_{t t}+D w_{x x} w_{x x t}-\frac{1}{2} N_{t}(t) w_{x}^{2}-N(t) w_{x} w_{x t}+\beta_{0} w w_{t}\right) d x .
\end{aligned}
$$

Проведем интегрирование с учетом уравнения (1) и граничных условий (2)-(4). Применяя формулу Грина, получим

$$
\begin{aligned}
& \iint_{G}\left(\varphi_{x} \varphi_{x t}+\varphi_{y} \varphi_{y t}\right) d x d y= \\
& \quad=\iint_{G}\left(\left(\varphi_{x} \varphi_{t}\right)_{x}+\left(\varphi_{y} \varphi_{t}\right)_{y}\right) d x d y-\iint_{G}\left(\varphi_{x x}+\varphi_{y y}\right) \varphi_{t} d x d y=\oint_{\partial G} \varphi_{x} \varphi_{t} d y-\oint_{\partial G} \varphi_{y} \varphi_{t} d x=
\end{aligned}
$$




$$
\begin{gathered}
=\int_{a}^{b} \varphi_{y}^{-}\left(x, h_{*}, t\right) \varphi_{t}^{-}\left(x, h_{*}, t\right) d x-\int_{a}^{b} \varphi_{y}^{+}\left(x, h_{*}, t\right) \varphi_{t}^{+}\left(x, h_{*}, t\right) d x= \\
=-\int_{a}^{b}\left(w_{t}(x, t)+V w_{x}(x, t)\right)\left(\varphi_{t}^{+}\left(x, h_{*}, t\right)-\varphi_{t}^{-}\left(x, h_{*}, t\right)\right) d x
\end{gathered}
$$

где $\partial G$ - граница двусвязной области $G$.

Для функции $w(x, t)$, удовлетворяющей уравнениям (6), (7), имеем

$$
\begin{aligned}
m w_{t t}(x, t)= & -D w_{x^{4}}(x, t)-N(t) w_{x x}(x, t)-\beta_{2} w_{x^{4} t}(x, t)-\beta_{1} w_{t}(x, t)-\beta_{0} w(x, t)+ \\
& +\rho\left(\varphi_{t}^{+}\left(x, h_{*}, t\right)+V \varphi_{x}^{+}\left(x, h_{*}, t\right)\right)-\rho\left(\varphi_{t}^{-}\left(x, h_{*}, t\right)+V \varphi_{x}^{-}\left(x, h_{*}, t\right)\right), \quad x \in(a, b) .
\end{aligned}
$$

Производная (9) согласно (10), (11) примет вид

$$
\begin{aligned}
& \Phi_{t}(t)=-2 \int_{a}^{b}\left(\varphi_{t}^{+}\left(x, h_{*}, t\right)-\varphi_{t}^{-}\left(x, h_{*}, t\right)\right) w_{t}(x, t) d x+ \\
& \quad+2 V \int_{a}^{b}\left(\varphi^{+}\left(x, h_{*}, t\right)-\varphi^{-}\left(x, h_{*}, t\right)\right) w_{x t}(x, t) d x+ \\
& +\frac{2}{\rho} \int_{a}^{b}\left(w _ { t } ( x , t ) \left(-D w_{x^{4}}(x, t)-N(t) w_{x x}(x, t)-\beta_{2} w_{x^{4} t}(x, t)-\beta_{1} w_{t}(x, t)-\beta_{0} w(x, t)+\right.\right. \\
& \left.\quad+\rho\left(\varphi_{t}^{+}\left(x, h_{*}, t\right)-\varphi_{t}^{-}\left(x, h_{*}, t\right)\right)+\rho V\left(\varphi_{x}^{+}\left(x, h_{*}, t\right)-\varphi_{x}^{-}\left(x, h_{*}, t\right)\right)\right)+ \\
& \left.+D w_{x x}(x, t) w_{x x t}(x, t)-\frac{1}{2} N_{t}(t) w_{x}^{2}(x, t)-N(t) w_{x}(x, t) w_{x t}(x, t)+\beta_{0} w(x, t) w_{t}(x, t)\right) d x
\end{aligned}
$$

Интегрируя по частям, согласно (5) получим

$$
\begin{aligned}
\int_{a}^{b}\left(\varphi^{+}\left(x, h_{*}, t\right)-\varphi^{-}\left(x, h_{*}, t\right)\right) w_{x t}(x, t) d x & =-\int_{a}^{b}\left(\varphi_{x}^{+}\left(x, h_{*}, t\right)-\varphi_{x}^{-}\left(x, h_{*}, t\right)\right) w_{t}(x, t) d x, \\
\int_{a}^{b} w_{t}(x, t) w_{x^{4}}(x, t) d x & =\int_{a}^{b} w_{x x t}(x, t) w_{x x}(x, t) d x, \\
\int_{a}^{b} w_{t}(x, t) w_{x^{4} t}(x, t) d x & =\int_{a}^{b} w_{x x t}^{2}(x, t) d x, \\
\int_{a}^{b} w_{t}(x, t) w_{x x}(x, t) d x & =\int_{a}^{b} w_{x t}(x, t) w_{x}(x, t) d x .
\end{aligned}
$$

После подстановки полученных равенств в (12), производная примет вид

$$
\Phi_{t}(t)=-\frac{2}{\rho} \int_{a}^{b}\left(\beta_{2} w_{x x t}^{2}+\beta_{1} w_{t}^{2}+\frac{1}{2} N_{t}(t) w_{x}^{2}\right) d x .
$$

Рассмотрим краевую задачу для уравнения $\psi_{x^{4}}(x)=\mu \psi(x)$ с краевыми условиями (5). Эта задача является самосопряженной и полностью определенной. Действительно, интегрируя по 
частям, нетрудно убедиться, что

$$
\begin{aligned}
& \int_{a}^{b} u_{x^{4}}(x) v(x) d x=\int_{a}^{b} u(x) v_{x^{4}}(x) d x, \quad \int_{a}^{b} u(x) v(x) d x=\int_{a}^{b} v(x) u(x) d x, \\
& \int_{a}^{b} u(x) u_{x^{4}}(x) d x>0, \quad \int_{a}^{b} u(x) u(x) d x>0
\end{aligned}
$$

для любых функций $u(x)$ и $v(x)$, удовлетворяющих рассматриваемым краевым условиям и имеющих на $[a, b]$ непрерывные производные четвертого порядка. Для функции $w_{t}(x, t)$ запишем неравенство Рэлея [14]:

$$
\int_{a}^{b} w_{x^{4} t}(x, t) w_{t}(x, t) d x \geqslant \mu_{1} \int_{a}^{b} w_{t}(x, t) w_{t}(x, t) d x,
$$

где $\mu_{1}=\pi^{4} /(b-a)^{4}$ - наименьшее собственное значение рассматриваемой краевой задачи. Интегрируя по частям, представим это неравенство в виде:

$$
\int_{a}^{b} w_{x x t}^{2}(x, t) d x \geqslant \frac{\pi^{4}}{(b-a)^{4}} \int_{a}^{b} w_{t}^{2}(x, t) d x .
$$

Подставляя (14) в (13), получим

$$
\Phi_{t}(t) \leqslant-\frac{2}{\rho} \int_{a}^{b}\left(\left(\frac{\beta_{2} \pi^{4}}{(b-a)^{4}}+\beta_{1}\right) w_{t}^{2}+\frac{1}{2} N_{t}(t) w_{x}^{2}\right) d x .
$$

Пусть выполняются условия

$$
\frac{\beta_{2} \pi^{4}}{(b-a)^{4}}+\beta_{1} \geqslant 0, \quad \beta_{0} \geqslant 0, N_{t}(t) \geqslant 0,
$$

тогда из $(15)$ получим оценку $\Phi_{t}(t) \leqslant 0$. Проинтегрируем по переменной $t$ от 0 до $t$ :

$$
\Phi(t) \leqslant \Phi(0)
$$

где

$$
\begin{aligned}
\Phi(0)=\iint_{G}\left(\varphi_{x}^{2}(x, y, 0)\right. & \left.+\varphi_{y}^{2}(x, y, 0)\right) d x d y+ \\
& +2 V \int_{a}^{b}\left(\varphi^{+}\left(x, h_{*}, 0\right)-\varphi^{-}\left(x, h_{*}, 0\right)\right) w_{x}(x, 0) d x+ \\
& +\frac{1}{\rho} \int_{a}^{b}\left(m w_{t 0}^{2}+D w_{x x 0}^{2}-N(0) w_{x 0}^{2}+\beta_{0} w_{0}^{2}\right) d x .
\end{aligned}
$$

Индекс 0 снизу в (18) обозначает значение функции при $t=0$.

Рассматривая краевую задачу для уравнения $\psi_{x^{4}}(x)=-\lambda \psi_{x^{2}}(x)$ с краевыми условиями (5), аналогично (14), получим

$$
\int_{a}^{b} w_{x x}^{2} d x \geqslant \frac{\pi^{2}}{(b-a)^{2}} \int_{a}^{b} w_{x}^{2} d x
$$


Согласно (8), (19) оценим $\Phi(t)$ снизу:

$$
\begin{aligned}
\Phi(t) \geqslant \iint_{G}\left(\varphi_{x}^{2}(x, y, t)\right. & \left.+\varphi_{y}^{2}(x, y, t)\right) d x d y+ \\
& +2 V \int_{a}^{b}\left(\varphi^{+}\left(x, h_{*}, t\right)-\varphi^{-}\left(x, h_{*}, t\right)\right) w_{x}(x, t) d x+ \\
& +\frac{1}{\rho} \int_{a}^{b}\left(\frac{\pi^{2} D}{(b-a)^{2}}-N(t)\right) w_{x}^{2} d x .
\end{aligned}
$$

Для оценки двойного интеграла разобьем область $G$ на две области

$$
G_{1}=\left\{(x, y) \in \mathbb{R}^{2}: 0<x<l, 0<y<h_{*}\right\}, \quad G_{2}=\left\{(x, y) \in \mathbb{R}^{2}: 0<x<l, h_{*}<y<h\right\} .
$$

С учетом граничных условий (2) согласно неравенству Коши-Буняковского получим

$$
\iint_{G_{1}} \varphi_{x}^{2} d x d y \geqslant \frac{\pi^{2}}{l^{2}} \iint_{G_{1}} \varphi^{2} d x d y, \quad \iint_{G_{2}} \varphi_{x}^{2} d x d y \geqslant \frac{\pi^{2}}{l^{2}} \iint_{G_{2}} \varphi^{2} d x d y .
$$

Складывая, получим

$$
\iint_{G} \varphi_{x}^{2} d x d y \geqslant \frac{\pi^{2}}{l^{2}} \iint_{G_{1}} \varphi^{2} d x d y+\frac{\pi^{2}}{l^{2}} \iint_{G_{2}} \varphi^{2} d x d y .
$$

Согласно неравенству Коши-Буняковского имеем

$$
\left(\int_{y}^{h_{*}} \varphi_{y} d y\right)^{2} \leqslant \int_{y}^{h_{*}} 1^{2} d y \int_{y}^{h_{*}} \varphi_{y}^{2} d y
$$

Следовательно,

$$
\left(\varphi^{-}\left(x, h_{*}, t\right)-\varphi(x, y, t)\right)^{2} \leqslant\left(h_{*}-y\right) \int_{y}^{h_{*}} \varphi_{y}^{2} d y \leqslant\left(h_{*}-y\right) \int_{0}^{h_{*}} \varphi_{y}^{2} d y .
$$

Интегрируя от 0 до $h_{*}$ по переменной $y$, получим

$$
\int_{0}^{h_{*}}\left(\varphi^{-}\left(x, h_{*}, t\right)-\varphi(x, y, t)\right)^{2} d y \leqslant \frac{h_{*}^{2}}{2} \int_{0}^{h_{*}} \varphi_{y}^{2} d y .
$$

Интегрируя от 0 до $l$ по переменной $x$, окончательно находим

$$
\iint_{G_{1}} \varphi_{y}^{2} d x d y \geqslant \frac{2}{h_{*}^{2}} \iint_{G_{1}}\left(\varphi^{-}\left(x, h_{*}, t\right)-\varphi(x, y, t)\right)^{2} d x d y
$$

Аналогично, применяя неравенство Коши-Буняковского, получим

$$
\iint_{G_{2}} \varphi_{y}^{2} d x d y \geqslant \frac{2}{\left(h-h_{*}\right)^{2}} \iint_{G_{2}}\left(\varphi(x, y, t)-\varphi^{+}\left(x, h_{*}, t\right)\right)^{2} d x d y .
$$

Применяя (21)-(23) для (20), получим неравенство

$$
\begin{aligned}
\Phi(t) \geqslant \iint_{G_{1}}( & \left.\frac{\pi^{2}}{l^{2}} \varphi^{2}(x, y, t)+\frac{2}{h_{*}^{2}}\left(\varphi^{-}\left(x, h_{*}, t\right)-\varphi(x, y, t)\right)^{2}\right) d x d y+ \\
& +\iint_{G_{2}}\left(\frac{\pi^{2}}{l^{2}} \varphi^{2}(x, y, t)+\frac{2}{\left(h-h_{*}\right)^{2}}\left(\varphi(x, y, t)-\varphi^{+}\left(x, h_{*}, t\right)\right)^{2}\right) d x d y+
\end{aligned}
$$




$$
+2 V \int_{a}^{b}\left(\varphi^{+}\left(x, h_{*}, t\right)-\varphi^{-}\left(x, h_{*}, t\right)\right) w_{x}(x, t) d x++\frac{1}{\rho} \int_{a}^{b}\left(\frac{\pi^{2} D}{(b-a)^{2}}-N(t)\right) w_{x}^{2} d x .
$$

Введем обозначение

$$
f(x, t)= \begin{cases}0, & x \in(0, a) \\ w_{x}(x, t), & x \in[a, b] \\ 0, & x \in(b, l)\end{cases}
$$

тогда из (24) получим неравенство

$$
\begin{aligned}
& \Phi(t) \geqslant \iint_{G_{1}}\left(\frac{2}{h_{*}^{2}}\left(\varphi^{-}\left(x, h_{*}, t\right)-\varphi(x, y, t)\right)^{2}+\frac{\pi^{2}}{l^{2}} \varphi^{2}(x, y, t)\right) d x d y+ \\
&+\iint_{G_{2}}\left(\frac{\pi^{2}}{l^{2}} \varphi^{2}(x, y, t)+\frac{2}{\left(h-h_{*}\right)^{2}}\left(\varphi^{+}\left(x, h_{*}, t\right)-\varphi(x, y, t)\right)^{2}\right) d x d y+ \\
&+2 V \int_{0}^{l}\left(\varphi^{+}\left(x, h_{*}, t\right)-\varphi^{-}\left(x, h_{*}, t\right)\right) f(x, t) d x+\frac{1}{\rho} \int_{0}^{l}\left(\frac{\pi^{2} D}{(b-a)^{2}}-N(t)\right) f^{2}(x, t) d x= \\
&=\iint_{G_{1}}\left[\left(\frac{\pi^{2}}{l^{2}}+\frac{2}{h_{*}^{2}}\right) \varphi^{2}(x, y, t)-\frac{4}{h_{*}^{2}} \varphi^{-}\left(x, h_{*}, t\right) \varphi(x, y, t)+\frac{2}{h_{*}^{2}}\left(\varphi^{-}\left(x, h_{*}, t\right)\right)^{2}+\right. \\
&\left.\quad+\frac{2 V}{h_{*}} \varphi^{-}\left(x, h_{*}, t\right) f(x, t)+\frac{\left(\pi^{2} D-N(t)(b-a)^{2}\right) \chi}{\rho h_{*}(b-a)^{2}} f^{2}(x, t)\right] d x d y+ \\
&+\iint_{G_{2}}\left[\left(\frac{\pi^{2}}{l^{2}}+\frac{2}{\left(h-h_{*}\right)^{2}}\right) \varphi^{2}(x, y, t)-\frac{4}{\left(h-h_{*}\right)^{2}} \varphi^{+}\left(x, h_{*}, t\right) \varphi(x, y, t)+\frac{2}{\left(h-h_{*}\right)^{2}}\left(\varphi^{+}\left(x, h_{*}, t\right)\right)^{2}+\right. \\
&\left.\quad+\frac{2 V(1-\chi)}{h-h_{*}} \varphi^{+}\left(x, h_{*}, t\right) f(x, t)+\frac{\left(\pi^{2} D-N(t)(b-a)^{2}\right)(1-\chi)}{\rho\left(h-h_{*}\right)(b-a)^{2}} f^{2}(x, t)\right] d x d y, \quad(25)
\end{aligned}
$$

где $\chi \in(0,1)$.

Введем обозначения

$$
\begin{gathered}
d_{11}^{(1)}=\frac{\pi^{2}}{l^{2}}+\frac{2}{h_{*}^{2}}, \quad d_{22}^{(1)}=\frac{2}{h_{*}^{2}}, \quad d_{12}^{(1)}=d_{21}^{(1)}=\frac{2}{h_{*}^{2}}, \\
d_{23}^{(1)}=d_{32}^{(1)}=\frac{V}{h_{*}}, \quad d_{33}^{(1)}=\frac{\left(\pi^{2} D-N(t)(b-a)^{2}\right) \chi}{\rho h_{*}(b-a)^{2}} .
\end{gathered}
$$

Рассмотрим квадратичную форму относительно $\varphi(x, y, t), \varphi^{-}\left(x, h_{*}, t\right), f(x, t)$ в $(25)$. Согласно критерию Сильвестра, запишем условия положительной определенности квадратичной формы

$$
\begin{gathered}
\Delta_{1}^{(1)}=d_{11}^{(1)}>0, \quad \Delta_{2}^{(1)}=d_{11}^{(1)} d_{22}^{(1)}-d_{12}^{(1)^{2}}=\frac{2 \pi^{2}}{l^{2} h_{*}^{2}}>0 \\
\Delta_{3}^{(1)}=d_{33}^{(1)} \Delta_{2}^{(1)}-d_{23}^{(1)} d_{11}^{2}>0 .
\end{gathered}
$$

Первые два условия выполняются. Неравенство (27) примет вид:

$$
N(t)<\frac{\pi^{2} D}{(b-a)^{2}}-\frac{V^{2} l^{2} \rho h_{*}}{2 \pi^{2} \chi}\left(\frac{\pi^{2}}{l^{2}}+\frac{2}{h_{*}^{2}}\right) .
$$


Введем обозначения

$$
\begin{gathered}
d_{11}^{(2)}=\frac{\pi^{2}}{l^{2}}+\frac{2}{\left(h-h_{*}\right)^{2}}, \quad d_{22}^{(2)}=d_{12}^{(2)}=\frac{2}{\left(h-h_{*}\right)^{2}}, \quad d_{23}^{(2)}=\frac{V}{h-h_{*}}, \\
d_{33}^{(2)}=\frac{\left(\pi^{2} D-N(t)(b-a)^{2}\right)(1-\chi)}{\rho\left(h-h_{*}\right)(b-a)^{2}} .
\end{gathered}
$$

Рассмотрим квадратичную форму относительно $\varphi(x, y, t), \varphi^{+}\left(x, h_{*}, t\right), f(x, t)$ в $(25)$. Согласно критерию Сильвестра, запишем условия положительной определенности квадратичной формы

$$
\begin{gathered}
\Delta_{1}^{(2)}=d_{11}^{(2)}>0, \quad \Delta_{2}^{(2)}=d_{11}^{(2)} d_{22}^{(2)}-d_{12}^{(2)^{2}}=\frac{2 \pi^{2}}{l^{2}\left(h-h_{*}\right)^{2}}>0, \\
\Delta_{3}^{(2)}=d_{33}^{(2)} \Delta_{2}^{(2)}-d_{23}^{(2)^{2}} d_{11}^{(2)}>0 .
\end{gathered}
$$

Первые два условия выполняются. Неравенство (30) примет вид:

$$
N(t)<\frac{\pi^{2} D}{(b-a)^{2}}-\frac{V^{2} l^{2} \rho\left(h-h_{*}\right)}{2 \pi^{2}(1-\chi)}\left(\frac{\pi^{2}}{l^{2}}+\frac{2}{\left(h-h_{*}\right)^{2}}\right) .
$$

Из неравенств (28), (31) найдем оптимальный параметр $\chi$, обеспечивающий наиболее широкую область значений параметров, входящих в условия (28), (31). Для этого приравняем их правые части

$$
\frac{h_{*}}{\chi}\left(\frac{\pi^{2}}{l^{2}}+\frac{2}{h_{*}^{2}}\right)=\frac{h-h_{*}}{1-\chi}\left(\frac{\pi^{2}}{l^{2}}+\frac{2}{\left(h-h_{*}\right)^{2}}\right) .
$$

Тогда находим

$$
\chi=\frac{\left(\pi^{2} h_{*}^{2}+2 l^{2}\right)\left(h-h_{*}\right)}{\pi^{2} h\left(h-h_{*}\right) h_{*}+2 l^{2} h} .
$$

Таким образом, получим условие

$$
N(t)<\frac{\pi^{2} D}{(b-a)^{2}}-\frac{V^{2} \rho h\left(\pi^{2}\left(h-h_{*}\right) h_{*}+2 l^{2}\right)}{2 \pi^{2} h_{*}\left(h-h_{*}\right)} .
$$

Используя метод Лагранжа, с учетом неравенства (28) оценим квадратичную форму в (25)

$$
F\left(\varphi(x, y, t), \varphi^{-}\left(x, y_{0}, t\right), f(x, t)\right) \geqslant \frac{\Delta_{3}^{(1)}}{\Delta_{2}^{(1)}} f^{2}(x, t) .
$$

Учитывая неравенство Коши-Буняковского

$$
w^{2}(x, t) \leqslant(b-a) \int_{a}^{b} w_{x}^{2}(x, t) d x
$$

из (25) получим

$$
\begin{aligned}
\Phi(t) \geqslant \iint_{G_{1}} \frac{\Delta_{3}^{(1)}}{\Delta_{2}^{(1)}} f^{2}(x, t) d x d y=\int_{0}^{l} \frac{\Delta_{3}^{(1)} h_{*}}{\Delta_{2}^{(1)}} f^{2}(x, t) d x & = \\
& =\int_{a}^{b} \frac{\Delta_{3}^{(1)} h_{*}}{\Delta_{2}^{(1)}} w_{x}^{2}(x, t) d x \geqslant \frac{\Delta_{3}^{(1)} h_{*}}{\Delta_{2}^{(1)}(b-a)} w^{2}(x, t) .
\end{aligned}
$$


Учитывая (17), (18), (34), получим неравенство

$$
\begin{aligned}
w^{2}(x, t) \leqslant \frac{\Delta_{2}^{(1)}(b-a)}{\Delta_{3}^{(1)} h_{*}}\left[\iint_{G}\left(\varphi_{x}^{2}(x, y, 0)+\varphi_{y}^{2}(x, y, 0)\right) d x d y+\right. & \\
& +2 V \int_{a}^{b}\left(\varphi^{+}\left(x, h_{*}, 0\right)\right. \\
& \left.-\varphi^{-}\left(x, h_{*}, 0\right)\right) w_{x}(x, 0) d x+ \\
& \left.+\frac{1}{\rho} \int_{a}^{b}\left(m w_{t 0}^{2}+D w_{x x 0}^{2}-N(0) w_{x 0}^{2}+\beta_{0} w_{0}^{2}\right) d x\right] .
\end{aligned}
$$

Согласно (35) имеет место следующая теорема.

Теорема 1. Пусть выполняются условия (16), (33). Тогда решение $w(x, t)$ задачи (1)-(7) устойчиво по отношения $\kappa$ возмущениям начальных данных $\varphi_{x}(x, y, 0), \varphi_{y}(x, y, 0), w_{t}(x, 0)$, $w(x, 0), w_{x}(x, 0), w_{x x}(x, 0)$.

4. Исследование динамики. Введем обозначения: $\varphi_{1}(x, y, t)$ - потенциал скорости газа в области $G_{1}, \varphi_{2}(x, y, t)$ - потенциал скорости газа в области $G_{2}$. Тогда исследование сформулированной выше задачи можно свести к изучению следующей задачи:

$$
\begin{aligned}
& \varphi_{k_{x x}}+\varphi_{k_{y y}}=0, \quad k=1,2, \\
& \varphi_{1 y}\left(x, h_{*}, t\right)=\varphi_{2 y}\left(x, h_{*}, t\right), \quad x \in(0, l), \\
& \varphi_{1 y}\left(x, h_{*}, t\right)=w_{t}+V w_{x}, \quad x \in(a, b), \\
& -\left.\rho\left(\varphi_{1 t}+V \varphi_{1 x}\right)\right|_{y=h_{*}}+\left.\rho\left(\varphi_{2 t}+V \varphi_{2 x}\right)\right|_{y=h_{*}}=\left\{\begin{array}{ll}
L(w), & x \in(a, b), \\
0, & x \in(0, a) \cup(b, l),
\end{array},\right. \\
& \varphi_{1}(0, y, t)=\varphi_{2}(0, y, t)=0, \quad \varphi_{1}(l, y, t)=\varphi_{2}(l, y, t)=0, \quad y \in(0, h), \\
& \varphi_{1 y}(x, 0, t)=0, \quad \varphi_{2 y}(x, h, t)=0, \quad x \in(0, l), \\
& w(a, t)=w_{x x}(a, t)=0, \quad w(b, t)=w_{x x}(b, t)=0, \quad t \geqslant 0, \\
& w(x, 0)=f_{1}(x), \quad w_{t}(x, 0)=f_{2}(x), \quad x \in(a, b) .
\end{aligned}
$$

Здесь оператор $L(w)$ задается формулой (7). Уравнение Лапласа (36) описывает течение газа в канале в модели идеальной несжимаемой среды; (37) - равенство нормальных составляющих скорости на линии $y=h_{*} ;(38),(41)$ - условия непротекания; (39) - уравнение, описывающее при $x \in(a, b)$ динамику пластины, а при $x \in(0, a) \cup(b, l)$ задающее равенство давлений на линии $y=h_{*} ;(40)$ - условия отсутствия возмущений на входе и выходе из канала (справедливо для достаточно длинного канала); (42), (43)-граничные и начальные условия. Линия $y=h_{*}$ является линией контактного разрыва.

Потенциалы скорости $\varphi_{1}$ и $\varphi_{2}$, описывающие движение газа в областях $G_{1}$ и $G_{2}$, представим функциями

$$
\begin{array}{ll}
\varphi_{1}(x, y, t)=\sum_{n=1}^{\infty} a_{n}(t) \sin \lambda_{n} x\left(e^{\lambda_{n} y}+e^{-\lambda_{n} y}\right), & \lambda_{n}=\frac{n \pi}{l}, \\
\varphi_{2}(x, y, t)=\sum_{n=1}^{\infty} b_{n}(t) \sin \lambda_{n} x\left(e^{\lambda_{n} y}+e^{-\lambda_{n} y} e^{2 \lambda_{n} h}\right), & \lambda_{n}=\frac{n \pi}{l} .
\end{array}
$$

Удовлетворяя условию (37), получим

$$
a_{n}(t)\left(e^{\lambda_{n} h_{*}}-e^{-\lambda_{n} h_{*}}\right)=b_{n}(t)\left(e^{\lambda_{n} h_{*}}-e^{-\lambda_{n} h_{*}} e^{2 \lambda_{n} h_{*}}\right) .
$$


Из условия (38) следует

$$
\sum_{n=1}^{\infty} a_{n}(t) \sin \lambda_{n} x\left(e^{\lambda_{n} h_{*}}-e^{-\lambda_{n} h_{*}}\right) \lambda_{n}=w_{t}+V w_{x}, \quad \lambda_{n}=\frac{n \pi}{l},
$$

Удовлетворяя условию (39), получим

$$
\begin{aligned}
-\sum_{n=1}^{\infty} a_{n}^{\prime}(t) \sin \lambda_{n} x\left(e^{\lambda_{n} h_{*}}+e^{-\lambda_{n} h_{*}}\right)-V \sum_{n=1}^{\infty} a_{n}(t) \lambda_{n} \cos \lambda_{n} x\left(e^{\lambda_{n} h_{*}}+e^{-\lambda_{n} h_{*}}\right)+ \\
+\sum_{n=1}^{\infty} b_{n}^{\prime}(t) \sin \lambda_{n} x\left(e^{\lambda_{n} h_{*}}+e^{-\lambda_{n} h_{*}} e^{2 \lambda_{n} h}\right)+V \sum_{n=1}^{\infty} b_{n}(t) \lambda_{n} \cos \lambda_{n} x\left(e^{\lambda_{n} h_{*}}+e^{-\lambda_{n} h_{*}} e^{2 \lambda_{n} h}\right)= \\
= \begin{cases}\frac{1}{\rho} L(w), & x \in(a, b), \\
0, & x \in(0, a) \cup(b, l) .\end{cases}
\end{aligned}
$$

Применим метод Галеркина. Функцию деформации $w(x, t)$ будем искать в виде

$$
w(x, t)=\sum_{k=1}^{\infty} w_{k}(t) \sin \mu_{k}(x-a), \quad \mu_{k}=\frac{k \pi}{b-a},
$$

где $\left\{\sin \mu_{k}(x-a)\right\}_{k=1}^{\infty}$ - полная система базисных функций на отрезке $[a, b]$, подобранных так, чтобы выполнялись заданные краевые условия (42).

Проецируя невязку уравнения (45) на систему функций $\left\{\sin \mu_{k}(x-a)\right\}_{k=1}^{\infty}$, невязку уравнения (46) на систему функций $\left\{\sin \lambda_{k} x\right\}_{k=1}^{\infty}$ и, пользуясь уравнением (44), получим систему обыкновенных дифференциальных уравнений для нахождения неизвестных функций $a_{n}(t), b_{n}(t), w_{n}(t)$ :

$$
\begin{gathered}
\sum_{n=1}^{\infty} a_{n}(t) 2 \lambda_{n} \operatorname{sh} \lambda_{n} h_{*} G_{m n}=\frac{b-a}{2} \dot{w}_{m}(t)+V \sum_{k=1}^{\infty} w_{k}(t) \sin \mu_{k} Q_{m k}, \quad m=1,2, \ldots \\
-a_{m}^{\prime}(t) l \operatorname{ch~} \lambda_{m} h_{*}+a_{m}^{\prime}(t) l e^{\lambda_{m} h} \operatorname{sh} \lambda_{m} h_{*} \operatorname{th} \lambda_{m}\left(h-h_{*}\right)- \\
-2 V \sum_{n=1}^{\infty} a_{n}(t) \lambda_{n} \operatorname{ch~} \lambda_{n} h_{*} H_{m n}+2 V \sum_{n=1}^{\infty} a_{n}(t) \lambda_{n} \operatorname{sh} \lambda_{n} h_{*} e^{\lambda_{n} h} H_{m n} \operatorname{cth} \lambda_{n}\left(h-h_{*}\right)= \\
=\frac{1}{\rho}\left(m \sum_{k=1}^{\infty} \ddot{w}_{k}(t) G_{k m}+D \sum_{k=1}^{\infty} w_{k}(t) \mu_{k}^{4} G_{k m}\right), \quad m=1,2, \ldots
\end{gathered}
$$

где $G_{m n}, H_{m n}, Q_{m n}$ - числовые коэффициенты, выражаемые через параметры задачи.

Применяя для начальных условий (43) метод Галеркина, получим начальные значения $w_{n}(0), \dot{w}_{n}(0)$. Полагая в начальный момент времени поток невозмущенным, можно положить $\varphi_{1}(x, y, 0)=0$, тогда $a_{n}(0)=0$.

Получаем, таким образом, задачу Коши для неизвестных функций $w_{n}(t), a_{n}(t)$. При численном решении этой задачи в выражениях для $\varphi_{1}(x, y, t), \varphi_{2}(x, y, t), w(x, t)$ берутся конкретные количества слагаемых.

5. Пример. Для численного решения задачи в выражениях для $\varphi_{1}(x, y, t), \varphi_{2}(x, y, t)$ и $w(x, t)$ ограничимся двумя слагаемыми для каждого выражения.

Будем считать, что упругий элемент изготовлен из алюминия $\left(E=7,1 \cdot 10^{10}\right.$ - модуль упругости, $\rho_{p l}=2770$ - плотность, $v=0,33$ - коэффициент Пуассона, $h_{p l}=0,002$ - толщина пластинки), резервуар заполнен эмульсией $(\rho=1100)$. Скорость потока $V=1$. Другие параметры механической системы: $l=2 ; h=0,5 ; h_{*}=0,25 ; a=0,9 ; b=1,1 ; m=5,54$ (погонная масса); $D=\frac{E h_{p l}^{3}}{12\left(1-v^{2}\right)}=53,1179$ (изгибная жесткость); $\beta_{0}=0,1 ; \beta_{1}=0,1 ; \beta_{2}=0,1 ; N(t)=-1000$. Все значения приведены в единицах СИ. Начальные условия зададим в виде

$$
w(x, 0)=0,001 \sin \mu_{1}(x-a), \quad w_{t}(x, 0)=0,005 \sin \mu_{1}(x-a) .
$$




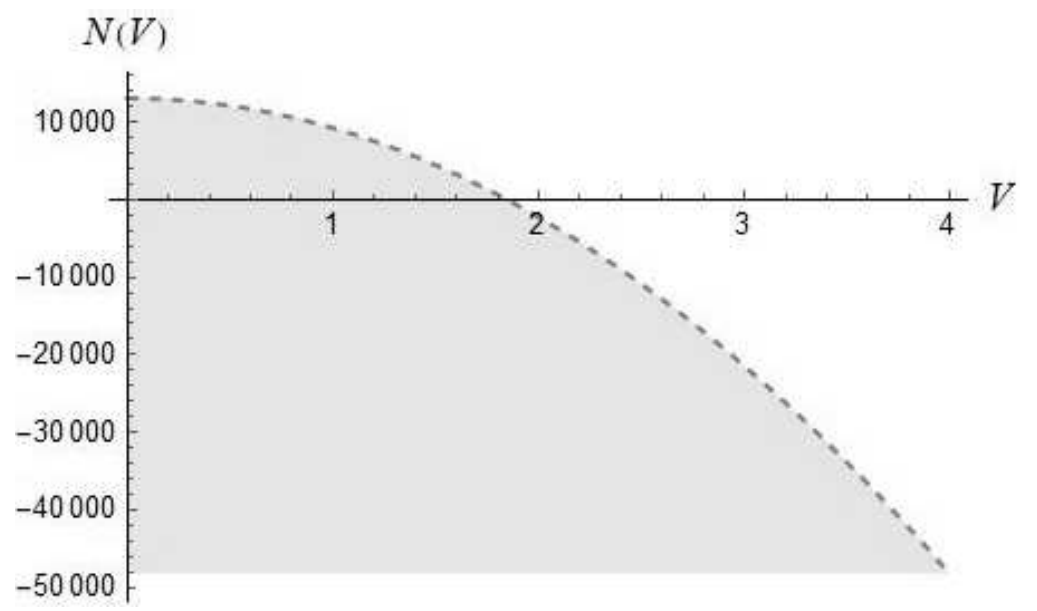

Рис. 2. Область устойчивости на плоскости $(V, N)$

Для указанных значений параметров построена область устойчивости (серая область) согласно неравенству (33) на плоскости $(V, N)$ (рис. 2).

На рис. 3 приведен график функции

$$
w\left(x_{0}, t\right)=\sum_{k=1}^{2} w_{k}(t) \sin \mu_{k}\left(x_{0}-a\right)
$$

в точке $x_{0}=(a+b) / 2,1$. Как видно из рис. 3 , колебания упругого элемента имеют устойчивый характер. Отметим, что точка $(V, N)=(1,-1000)$ попадает в область устойчивости, изображенную на рис. 2.

Для указанных выше значений параметров изменим величину усилия $N(t)=10000$ и скорости потока $V=10$. Получим график функции $w\left(x_{0}, t\right)$ в точке $x_{0}=(a+b) / 2,1$ (см. pис. 4$)$ :

Как видно из рис. 4 , колебания упругого элемента имеют неустойчивый характер. Отметим, что точка $(V, N)=(10,10000)$ находится вне области устойчивости, изображенной на рис. 2 .

Рассматривалась также нелинейная модель, учитывающая демпфирование продольного усилия и изгибающего момента упругих элементов [12]. В этом случае динамика упругих элементов описывается уравнениями для продольной и поперечной деформаций:

$$
\begin{gathered}
m u_{t t}(x, t)-G_{x}(x, t)-\tau G_{x t}(x, t)+f\left(x, t, u, w, u_{t}, w_{t}\right)=0, \\
m w_{t t}(x, t)-\left[w _ { x } ( x , t ) \left(\begin{array}{l}
\left.\left.G(x, t)+\tau G_{t}(x, t)\right)\right]_{x}+ \\
+M_{x x}(x, t)+\gamma M_{t x x}(x, t)+g\left(x, t, u, w, u_{t}, w_{t}\right)= \\
=\rho\left(\phi_{t}^{+}+V \phi_{x}^{+}\right)-\rho\left(\phi_{t}^{-}+V \phi_{x}^{-}\right), \quad x \in(a, b) .
\end{array}\right.\right.
\end{gathered}
$$

Здесь

$$
G(x, t)=E F\left(u_{x}+\frac{1}{2} w_{x}^{2}\right), \quad M(x, t)=E J \frac{w_{x x}}{\left[1+2\left(u_{x}+\frac{1}{2} w_{x}^{2}\right)\right]^{3 / 2}}
$$

- продольное усилие и изгибающий момент; $E F$-жесткость на растяжение; $E J$-жесткость на изгиб; $f\left(x, t, u, w, u_{t}, w_{t}\right), g\left(x, t, u, w, u_{t}, w_{t}\right)$ - функции, описывающие некоторые внешние (например, управляющие) воздействия; $\tau, \gamma$ - коэффициенты демпфирования на растяжение и изгиб. Полагая прогибы малыми, можно положить $M(x, t)=E J\left[w_{x x}-3\left(u_{x}+\frac{1}{2} w_{x}^{2}\right) w_{x x}\right]$ или $M(x, t)=E J w_{x x}$. 


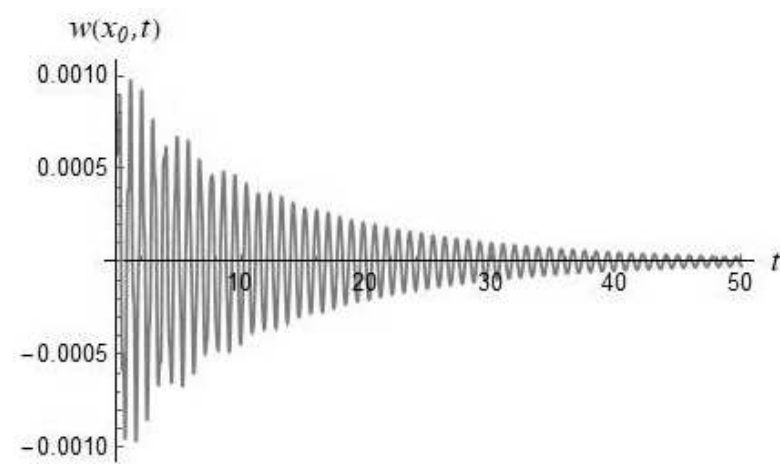

Рис. 3. График функции

$$
w\left(x_{0}, t\right)=\sum_{k=1}^{2} w_{k}(t) \sin \mu_{k}\left(x_{0}-a\right)
$$

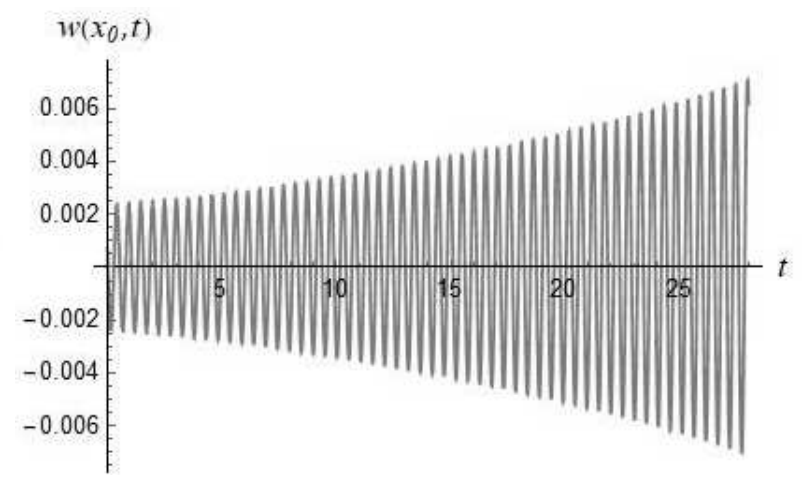

Рис. 4. График функции

$$
w\left(x_{0}, t\right)=\sum_{k=1}^{2} w_{k}(t) \sin \mu_{k}\left(x_{0}-a\right)
$$

6. Заключение. На основе предложенной математической модели колебаний упругого элемента вибрационного устройства в виде пластины-полосы, обтекаемой дозвуковым потоком идеальной жидкости, проведено исследование динамики и динамической устойчивости этого элемента. Модель описывается связанной системой дифференциальных уравнений с частными производными для деформации элемента и потенциала скорости жидкости. С помощью построенного функционала получены достаточные условия устойчивости решений этой системы уравнений. Полученные условия устойчивости накладывают ограничения на изгибную жесткость элемента, сжимающее (растягивающее) элемент усилие, скорость невозмущенного однородного потока, а также на коэффициенты внутреннего и внешнего демпфирования. Эти условия явно содержат основные параметры механической системы, и в таком виде они наиболее приспособлены для решения задач оптимизации, автоматического управления, автоматизированного проектирования. Разработан также численный метод исследования динамики упругого элемента вибрационного устройства, на основе которого проведен численный эксперимент.

Методы исследования устойчивости и динамики упругих элементов вибрационных устройств, предлагаемые в данной статье, можно применить в случае произвольного количества упругих элементов, расположенных последовательно друг за другом $\left(x \in\left(a_{k}, b_{k}\right), k=1, \ldots, n ; y=h_{*}\right)$ или параллельно друг над другом $\left(x \in(a, b) ; y=h_{k}, k=1, \ldots, n\right)$.

\section{СПИСОК ЛИТЕРАТУРЫ}

1. Агеев Р. В., Могилевич Л. И., Попов В. С. Колебания стенок щелевого канала с вязкой жидкостью, образованного трехслойным и твердым дисками// Пробл. машиностр. надежн. машин. $-2014 .-1$. - C. 3-11.

2. Агеев Р. В., Могилевич Л. И., Попов В. С., Кузнецова Е. Л., Куликов Н. И. Математическая модель движения пульсирующего слоя вязкой жидкости в канале с упругой стенкой// Вестн. Перм. нац. иссл. политехн. ун-та. Мех. - 2014. - 3. - С. 17-35.

3. Агеев Р. В., Могилевич Л. И., Попов В. С., Попова А. А. Движение вязкой жидкости в плоском канале, образованном вибрирующим штампом и шарнирно опертой пластиной// Тр. МАИ. - 2014. 78. - C. $1-13$.

4. Анкилов А. В., Вельмисов П. А. Устойчивость решений некоторых классов интегро-дифференциальных уравнений в частных производных// Вестн. Самар. гос. ун-та. - 2008. - 8/1 (67). - С. 331-344.

5. Анкилов А. В., Вельмисов П. А. Динамика и устойчивость упругих пластин при аэрогидродинамическом воздействии. - Ульяновск: УлГТУ, 2009.

6. Анкилов А. В., Вельмисов П. А. Математическое моделирование в задачах динамической устойчивости деформируемых элементов конструкций при аэрогидродинамическом воздействии. - Ульяновск: УлГТУ, 2013.

7. Анкилов А. В., Вельмисов П. А. Функционалы Ляпунова в некоторых задачах динамической устойчивости аэроупругих конструкций. - Ульяновск: УлГТУ, 2015. 
8. Анкилов А. В., Вельмисов П. А., Семенова Е. П. Исследование динамической устойчивости упругих элементов стенок канала// Вестн. Саратов. гос. техн. ун-та. - 2009. - 2 (38), № 1. - С. 7-17.

9. Вельмисов П. А., Анкилов А. В. Динамическая устойчивость деформируемых элементов конструкций при сверхзвуковом режиме обтекания// Вестн. Самар. гос. техн. ун-та. Сер. Физ.-мат. науки. - 2018. -22 , № 1 . - C. 96-115.

10. Вельмисов П. А., Покладова Ю. В. Исследование динамики деформируемых элементов некоторых аэрогидроупругих систем. - Ульяновск: УлГТУ, 2018.

11. Вельмисов П. А., Покладова Ю. В. Математическое моделирование динамики упругих элементов, взаимодействующих с потоком газа// Вестн. Ульяновск. гос. техн. ун-та. - 2018. - 3. - С. $22-30$.

12. Вельмисов П. А., Покладова Ю. В. Математическое моделирование динамики защитной поверхности резервуара// Вестн. Ульяновск. гос. техн. ун-та. - 2018. - 2. - С. 27-35.

13. Вельмисов П. А., Покладова Ю. В., Серебрянникова Е. С. Математическое моделирование системы «трубопровод-датчик давления» // Ж. Средневолж. мат. о-ва. - 2010. - 12, № 4. - С. 85-93.

14. Коллати, Л. Задачи на собственные значения. - М.: Наука, 1968.

15. Куликов A. Н. Бифуркация автоколебаний при малом коэффициенте демпфирования в сверхзвуковом потоке газа// Прикл. мат. мех. - 2009. - 73, № 2. - С. 271-281.

16. Могилевич Л. И., Попов В. С., Попова А. А., Христофорова А. В. Математическое моделирование динамики взаимодействия сильновязкой жидкости со стенками канала, установленного на упругом основании // Динам. сист. мех. машин. - 2016. - 3, № 1. - С. 350-354.

17. Abdelbaki A. R., Paidoussis M. P., Misra A. K. A nonlinear model for a free-clamped cylinder subjected to confined axial flow// J. Fluids Struct. - 2018. - 80. - P. 390-404.

18. Abdelbaki A. R., Paidoussis M. P., Misra A. K. A nonlinear model for a hanging tubular cantilever simultaneously subjected to internal and confined external axial flows// J. Sound Vibr. — 2019. — 449. — P. 349-367.

19. Ankilov A. V., Velmisov P. A. Mathematical modelling of dynamics and stability of elastic elements of vibration devices// IFAC-PapersOnLine. — 2015. — 48, № 11. - P. 970-975.

20. Ankilov A. V., Vel'misov P. A. Stability of solutions to an aerohydroelasticity problem// J. Math. Sci. 2016. - 219, № 1. - P. 14-26.

21. Faal R. T., Derakhshan D. Flow-induced vibration of pipeline on elastic support// Proc. Eng. — 2011. 14. - P. 2986-2993.

22. Gatica G. N., Heuer N., Meddahi S. Coupling of mixed finite element and stabilized boundary element methods for a fluid-solid interaction problem in 3D// Num. Meth. Partial Differ. Equations. — 2014. 30, № 4. - P. 1211-1233.

23. Kontzialis K., Moditis K., Paidoussis M. P. Transient simulations of the fluid-structure interaction response of a partially confined pipe under axial flows in opposite directions// J. Pressure Vessel Techn. — 2017. — 139 (3). - P. 1-8.

24. Moditis K., Paidoussis M., Ratigan J. Dynamics of a partially confined, discharging, cantilever pipe with reverse external flow// J. Fluids Struct. - 2016. - 63. - P. 120-139.

25. Mogilevich L. I., Popova A. A., Popov V. S. On the dynamic interaction of an elastic cylindrical shell with a fluid laminar stream inside in application to pipeline transportation// Sci. Techn. Transport. — 2007. — 2. - P. 69-72.

26. Moshkelgosha E., Askari E., Jeong K. H., Shafiee A. A. Fluid-structure coupling of concentric double FGM shells with different lengths// J. Struct. Eng. Mech. — 2017. — 61, № 2. — P. 231-244.

Вельмисов Петр Александрович

Ульяновский государственный технический университет

E-mail: velmisov@ulstu.ru

Анкилов Андрей Владимирович

Ульяновский государственный технический университет

E-mail: ankil@ulstu.ru

Покладова Юлия Валерьевна

Ульяновский государственный технический университет

E-mail: pokladovau@inbox.ru 